\title{
Momentum-resolved radio-frequency spectroscopy of a spin-orbit coupled atomic Fermi gas near a Feshbach resonance in harmonic traps
}

\author{
Shi-Guo Peng ${ }^{1}$, Xia-Ji $\mathrm{Liu}^{2}$, Hui $\mathrm{Hu}^{2}$, and Kaijun Jiang, ${ }^{1,3}$ 雨 \\ ${ }^{1}$ State Key Laboratory of Magnetic Resonance and Atomic and Molecular Physics, \\ Wuhan Institute of Physics and Mathematics, Chinese Academy of Sciences, Wuhan 430071, China \\ ${ }^{2}$ ARC Centre of Excellence for Quantum-Atom Optics, \\ Centre for Atom Optics and Ultrafast Spectroscopy, \\ Swinburne University of Technology, Melbourne 3122, Australia and \\ ${ }^{3}$ Center for Cold Atom Physics, Chinese Academy of Sciences, Wuhan 430071, China
}

(Dated: June 1, 2018)

\begin{abstract}
We theoretically investigate the momentum-resolved radio-frequency spectroscopy of a harmonically trapped atomic Fermi gas near a Feshbach resonance in the presence of equal Rashba and Dresselhaus spin-orbit coupling. The system is qualitatively modeled as an ideal gas mixture of atoms and molecules, in which the properties of molecules, such as the wavefunction, binding energy and effective mass, are determined from the two-particle solution of two-interacting atoms. We calculate separately the radio-frequency response from atoms and molecules at finite temperatures by using the standard Fermi golden rule, and take into account the effect of harmonic traps within local density approximation. The total radio-frequency spectroscopy is discussed, as functions of temperature and spin-orbit coupling strength. Our results give a qualitative picture of radio-frequency spectroscopy of a resonantly interacting spin-orbit coupled Fermi gas and can be directly tested in atomic Fermi gases of ${ }^{40} \mathrm{~K}$ atoms at Shanxi University and of ${ }^{6} \mathrm{Li}$ atoms at MIT.
\end{abstract}

\section{INTRODUCTION}

Thanks to the high-controllability of ultracold atoms in interatomic interaction, geometry, purity, atomic species and lattice constant (of optical lattices), ultracold atomic gases have already become one of the most important footstones in modern physics, and can be used in simulating various strongly correlated many-body models in solid state physics [1] 3]. Very recently, another controllability of ultracold atoms is realized experimentally, in which the spin degree of freedom of atoms (i.e., the atomic internal hyperfine states) is coupled to the orbital degree of freedom (i.e., the momentum) by using a two-photon Raman process. This so-called spin-orbit (SO) coupling was first created and detected in atomic ${ }^{87} \mathrm{Rb}$ Bose-Einstein condensates (BEC) [4, 5], and then produced in atomic Fermi gases of ${ }^{40} \mathrm{~K}$ atoms [ [6] and ${ }^{6} \mathrm{Li}$ atoms [7]. The realization of $\mathrm{SO}$ coupled atomic gases enables the simulation of charged particles by using neutral atoms, which are cleaner and more controllable [8]. These experiments open an entirely new way to study the celebrated effects of SO coupling, such as topological insulators, topological superconductors and new exotic superfluid phases [9-11].

Radio-frequency (rf) spectroscopy has been widely applied in many experiments to study fermionic pairing in a two-component Fermi gas near Feshbach resonances when it crosses from a Bardeen-Cooper-Schrieffer (BCS) superfluid of weakly interacting Cooper pairs over to a BEC of tightly bound molecules [12, 13], and also to investigate the properties of polarons in spin-imbalanced

*Electronic address: kjjiang@wipm.ac.cn
Fermi gases [14 17]. This technology allows a precise determination of the molecular binding energy [12, 18] and pairing gap in a degenerate Fermi gas [12]. In addition, the momentum-resolved rf spectroscopy, i.e., the spectroscopy at a specific momentum, is also available [19] and provides important information on low-energy excitations of novel exotic state of matter in ultracold atoms. In this respect, it would be a powerful tool for characterizing the recently realized spin-orbit coupled atomic Fermi gases. Indeed, momentum-resolved rf spectroscopy of non-interacting spin-orbit coupled ${ }^{40} \mathrm{~K}$ and ${ }^{6} \mathrm{Li}$ atoms has already been reported [6, 7].

In this work, we aim to qualitatively predict the momentum-resolved rf spectroscopy of a resonantly interacting atomic Fermi gas with equal Rashba and Dresselhaus spin-orbit coupling, a system already realized experimentally at Shanxi University and also at MIT by using broad Feshbach resonances [20, 21]. As is wellknown, a strongly interacting Fermi gas near Feshbach resonances is notoriously difficult to handle theoretically [22, 23]. Therefore, at the qualitative level, we approximate the strongly interacting Fermi gas as a mixture of non-interacting atoms and molecules. All the properties of individual molecules are determined from the two-particle solution of two-interacting atoms. Our approximation may be justified at large temperatures well above the superfluid transition temperature $T_{c}$, where molecules are formed below the Feshbach resonance and have little correlations among themselves or with atoms. This is exactly the situation in current experiments, for instance, at Shanxi University [6], the typical temperature of spin-orbit coupled ${ }^{40} \mathrm{~K}$ atoms is now at about $0.6 T_{F}$, where $T_{F}$ is the Fermi degenerate temperature.

We consider separately the momentum-resolved rfspectroscopy of atoms and of molecules. Moreover, ac- 
cording to real experiments we take into account the crucial trapping potential that prevents the atoms and molecules from escaping. Within local density approximation (LDA), the trapped Fermi cloud may be divided into many locally uniform subsystems. For each subsystem, we calculate the rf responses of the atomic and molecular components based on the one-particle and two-particle solutions of uniform systems and Fermi's golden rule. We finally add up all the local contributions to determine the total momentum-resolved rf spectroscopy. We note that due to SO coupling, weakly bound molecules with anisotropic mass and wavefunction may be formed [24 26]. The bound to free rf transition of weakly bound molecules at rest has been predicted [27]. In this work, we will consider the rf transition of weakly bound molecules at motion with arbitrary center-of-mass (COM) momentum.

The paper is arranged as follows. In the next section, we consider the situation of current experiments, and introduce the model Hamiltonian responsible for equal Rashba and Dresselhaus SO coupling. In addition, we introduce the LDA formulism and present the calculation for the chemical potential. Then, the single-particle and two-particle problems of a local uniform subsystem are solved in Sec. III. The general formulas for the binding energy and wavefunction of two-particle bound state with non-zero COM momentum are also provided. In Sec. [V] we derive the momentum-resolved rf transition signals for non-interacting atoms and molecules, respectively, and then obtain the total $\mathrm{rf}$ spectroscopy for the harmonically trapped ideal gas mixture of fermionic atoms and bosonic molecules. Finally, our main results are concluded in Sec $\mathrm{V}$

\section{MODELS}

\section{A. Hamiltonian}

We consider a SO coupled Fermi gas with atomic mass $m$ in a harmonic trap $V(\mathbf{r})=m\left(\omega_{x}^{2} x^{2}+\omega_{y}^{2} y^{2}+\omega_{z}^{2} z^{2}\right) / 2$. The SO coupling is created by a two-photon Raman process [6, 7]. The many-body Hamiltonian responsible for this process may be modeled as $\mathcal{H}=\mathcal{H}_{0}+\mathcal{H}_{\text {int }}$, where

$$
\begin{aligned}
\mathcal{H}_{0}= & \sum_{\sigma} \int d \mathbf{r} \Psi_{\sigma}^{\dagger}(\mathbf{r})\left[-\frac{\hbar^{2} \nabla^{2}}{2 m}+V(\mathbf{r})-\mu\right] \Psi_{\sigma}(\mathbf{r})+ \\
& \int d \mathbf{r}\left[\Psi_{\uparrow}^{\dagger}(\mathbf{r})\left(\frac{\Omega_{R}}{2} e^{+i 2 k_{R} x}\right) \Psi_{\downarrow}(\mathbf{r})+H . c .\right]
\end{aligned}
$$

is the single-particle Hamiltonian and

$$
\mathcal{H}_{i n t}=U_{0} \int d \mathbf{r} \Psi_{\uparrow}^{\dagger}(\mathbf{r}) \Psi_{\downarrow}^{\dagger}(\mathbf{r}) \Psi_{\downarrow}(\mathbf{r}) \Psi_{\uparrow}(\mathbf{r})
$$

is the interaction Hamiltonian describing the contact interaction between two spin states. Here, $\Psi_{\sigma}^{\dagger}(\mathbf{r})$ is the creation field operator for atoms in the spin-state $\sigma$.
The second term in $\mathcal{H}_{0}$ describes the Raman coupling between two spin states with strength $\Omega_{R}, k_{R}$ is the wave-number of two Raman laser beams and therefore $2 \hbar k_{R}$ is the momentum transfer during the two-photon Raman process [6, 7]. The interaction strength is denoted by the bare interaction parameter $U_{0}$, which should be regularized in terms of the $s$-wave scattering length $a_{s}$, i.e., $1 / U_{0}=m /\left(4 \pi \hbar^{2} a_{s}\right)-(1 / V) \sum_{\mathbf{k}} m /\left(\hbar^{2} k^{2}\right)$. By using Feshbach resonances, the $s$-wave scattering length $a_{s}$ could be arbitrarily tuned, and the system may undergo a crossover from a BCS superfluid of weakly interacting Cooper pairs to a BEC of tightly bound molecules. Therefore, the atomic chemical potential $\mu$ may decrease as a result of decreasing atomic population, when weakly bound molecules are formed.

As argued in the Introduction section, we will treat the Fermi cloud as a mixture of non-interacting atoms and molecules, although the degree of freedom of molecules is not made explicitly in our model Hamiltonian from the outset. We will take the molecule as a two-particle bound state and determine all its properties by using two-particle solution of two interacting atoms. In the harmonic trap, we assume that these molecules experience the same harmonic trap as atoms, but with double mass, i.e., $V_{M}(\mathbf{r})=m\left(\omega_{x}^{2} x^{2}+\omega_{y}^{2} y^{2}+\omega_{z}^{2} z^{2}\right)$. Note that, our treatment of ideal mixture of atoms and molecules becomes exact for a Feshbach resonance with zero resonance width. However, for the broad Feshbach resonance used experimentally, this treatment is valid at the qualitative level only.

\section{B. Local density approximation}

If the number of atoms is sufficiently large, it is reasonable to assume that the trapped cloud may be divided into many locally uniform subsystems with a local chemical potential [28, 29]. Then, within LDA, the external trap $V(\mathbf{r})$ in the Hamiltonian (1) is absorbed into the chemical potential, and we can define effective local chemical potential

$$
\mu(\mathbf{r})=\mu-\frac{1}{2} m\left(\omega_{x}^{2} x^{2}+\omega_{y}^{2} y^{2}+\omega_{z}^{2} z^{2}\right) .
$$

Note that the global chemical potential $\mu$, which is determined by the total number of atoms, can be regarded as the local chemical potential in the trap center $(\mathbf{r}=0)$.

In order to evaluate the chemical potential $\mu$, let us consider the ideal gas mixture of fermionic atoms and bosonic molecules at finite temperatures. The distributions of the atoms and molecules are respectively determined by the Fermi-Dirac and Bose-Einstein distributions,

$$
\begin{gathered}
f_{A}(\epsilon)=\frac{1}{e^{(\epsilon-\mu) /\left(k_{B} T_{A}\right)}+1}, \\
f_{M}(\epsilon)=\frac{1}{e^{\left(\epsilon-\mu_{M}\right) /\left(k_{B} T_{M}\right)}-1},
\end{gathered}
$$


where $k_{B}$ is Boltzmann's constant, and $T_{A, M}, \mu$ and $\mu_{M}$ are the temperatures, chemical potentials of atoms and molecules, respectively. From the thermal and chemical equilibrium conditions, we have the following relations, $T_{A}=T_{M} \equiv T$ and $\mu_{M}=2 \mu+\varepsilon_{B}$, where $\varepsilon_{B}$ is the binding energy of the molecules relative to the threshold of two free atoms. To ease the numerical workload, we will approximate it as $\varepsilon_{B} \simeq E_{B} \equiv \hbar^{2} /\left(m a_{s}^{2}\right)$. Here, $E_{B}$ is the binding energy in the absence of SO coupling.

The populations of the atoms $N_{A}$ (in a single spin component) and noncondensed molecules $N_{M}$ are given by

$$
\begin{gathered}
N_{A}=\int_{0}^{\infty} d \epsilon \rho_{A}(\epsilon) f_{A}(\epsilon), \\
N_{M}=\int_{0}^{\infty} d \epsilon \rho_{M}(\epsilon) f_{M}(\epsilon),
\end{gathered}
$$

where $\rho_{A, M}(\epsilon)$ are the density of states (DOS) of the atoms and molecules, respectively. Qualitatively, for atoms we shall use the well-known expression for DOS in harmonically trapped systems without SO coupling,

$$
\rho_{A}(\epsilon) d \epsilon=\frac{\epsilon^{2}}{2(\hbar \omega)^{3}} d \epsilon,
$$

where $\omega \equiv\left(\omega_{x} \omega_{y} \omega_{z}\right)^{1 / 3}$. For molecules, we instead use,

$$
\rho_{M}(\epsilon) d \epsilon=\frac{\sqrt{\gamma} \epsilon^{2}}{2(\hbar \omega)^{3}} d \epsilon .
$$

The factor $\sqrt{\gamma}$ appearing in the DOS of molecules is due to the SO coupling, which induces an effective mass $M_{x}=$ $\gamma \cdot(2 m)$ as we will make clear later. Combining Eqs. (4) and (5) and (6)-(9), the populations of the atoms and noncondensed molecules are given by

$$
\begin{aligned}
& N_{A}=-\left(\frac{k_{B} T}{\hbar \omega}\right)^{3} L i_{3}\left(-z_{A}\right), \\
& N_{M}=\sqrt{\gamma}\left(\frac{k_{B} T}{\hbar \omega}\right)^{3} L i_{3}\left(z_{M}\right),
\end{aligned}
$$

where $z_{A}=e^{\mu /\left(k_{B} T\right)}$ and $z_{M}=e^{\left(2 \mu+E_{B}\right) /\left(k_{B} T\right)}$ are the fugacities of the atoms and molecules, respectively, and $L i_{n}(z)$ is the polylogarithm function. Therefore, the chemical potential $\mu$ should satisfy the following equation,

$$
2 N_{A}+2\left[N_{M}+N_{M}^{(0)}\right]=N,
$$

where $N_{M}^{(0)}$ is the population of the condensed molecules, and $N$ is the total number of atoms. Note that one molecule is counted as two atoms. Above the critical temperature, i.e., $T>T_{c}$, there is no condensed molecules $\left(N_{M}^{(0)}=0\right)$, therefore Eqs. (10)-(12) yield

$$
-2 L i_{3}\left(-z_{A}\right)+2 \sqrt{\gamma} L i_{3}\left(z_{M}\right)=N\left(\frac{\hbar \omega}{k_{B} T}\right)^{3} .
$$

Then from Eq. (13), the chemical potential $\mu$ can numerically be evaluated at a specific temperature and interaction strength $E_{B}$. The molecule BEC occurs below the critical temperature, i.e., $T<T_{c}$. In this case, the chemical potential of molecules vanishes, i.e., $\mu_{M}=0$, which in turn gives $\mu=-E_{B} / 2$.

With the chemical potential $\mu$ obtained, the properties of the trapped systems may be calculated by integrating over the whole space, based on the local solutions of uniform systems. In the following sections, we will firstly solve single-particle and two-particle problems in uniform systems, and then investigate the rf spectroscopy of trapped gases under the LDA approximation.

\section{SINGLE- AND TWO-PARTICLE PROBLEMS FOR UNIFORM SYSTEMS}

\section{A. Single-particle solution}

The single-particle problem in a uniform system has been discussed in detail in our previous work [27, 30]. Here, for self-containment we summarize briefly the results. We focus on the following non-interacting Hamiltonian for the subsystem,

$$
\begin{aligned}
\mathcal{H}_{0}= & \sum_{\sigma} \int d \mathbf{r} \Psi_{\sigma}^{\dagger}(\mathbf{r}) \frac{\hbar^{2} \hat{\mathbf{k}}^{2}}{2 m} \Psi_{\sigma}(\mathbf{r})+ \\
& \frac{\Omega_{R}}{2} \int d \mathbf{r}\left[\Psi_{\uparrow}^{\dagger}(\mathbf{r}) e^{i 2 k_{R} x} \Psi_{\downarrow}(\mathbf{r})+\text { H.c. }\right],
\end{aligned}
$$

where $\hat{\mathbf{k}} \equiv-i \nabla$. Under the transformation [27, 30], $\Psi_{\uparrow}(\mathbf{r})=e^{+i k_{R} x} \psi_{\uparrow}(\mathbf{r})$ and $\Psi_{\downarrow}(\mathbf{r})=e^{-i k_{R} x} \psi_{\downarrow}(\mathbf{r})$, the spatial dependence of the Raman coupling term in Eq.(14) is removed and yields

$$
\begin{aligned}
\mathcal{H}_{0}= & \int d \mathbf{r} \psi_{\uparrow}^{\dagger}(\mathbf{r})\left[\frac{\hbar^{2}\left(k^{2}+k_{R}^{2}\right)}{2 m}+\lambda k_{x}\right] \psi_{\uparrow}(\mathbf{r})+ \\
& \int d \mathbf{r} \psi_{\downarrow}^{\dagger}(\mathbf{r})\left[\frac{\hbar^{2}\left(k^{2}+k_{R}^{2}\right)}{2 m}-\lambda k_{x}\right] \psi_{\downarrow}(\mathbf{r})+ \\
& h \int d \mathbf{r}\left[\psi_{\uparrow}^{\dagger}(\mathbf{r}) \psi_{\downarrow}(\mathbf{r})+\text { H.c. }\right]
\end{aligned}
$$

where $\mathbf{e}_{x}$ is the unit vector along $x$ direction. For convenience, the effective Zeeman field $h \equiv \Omega_{R} / 2$ and the SO coupling strength $\lambda \equiv \hbar^{2} k_{R} / m$ are introduced. This single-particle Hamiltonian (15) can be easily diagonalized to yield two eigenvalues [27, 30],

$$
E_{\mathbf{k} \pm}=\frac{\hbar^{2} k_{R}^{2}}{2 m}+\frac{\hbar^{2} k^{2}}{2 m} \pm \sqrt{h^{2}+\lambda^{2} k_{x}^{2}} .
$$

The symbols " \pm " stands for the two helicity branches, and the corresponding single-atom eigenstates in helicity basis take the form

$$
\left[\begin{array}{l}
|\mathbf{k}+\rangle \\
|\mathbf{k}-\rangle
\end{array}\right]=\left[\begin{array}{cc}
\cos \theta_{\mathbf{k}} & \sin \theta_{\mathbf{k}} \\
-\sin \theta_{\mathbf{k}} & \cos \theta_{\mathbf{k}}
\end{array}\right]\left[\begin{array}{l}
|\mathbf{k} \uparrow\rangle \\
|\mathbf{k} \downarrow\rangle
\end{array}\right]
$$


where $\theta_{\mathbf{k}}=\arctan \left[\left(\sqrt{h^{2}+\lambda^{2} k_{x}^{2}}-\lambda k_{x}\right) / h\right]$. It is obvious that the lowest single-particle energy occurs at $k_{\perp}=\sqrt{k_{y}^{2}+k_{z}^{2}}=0$ and $k_{x}=\sqrt{m^{2} \lambda^{2} / \hbar^{4}-h^{2} / \lambda^{2}}$ if $h<m \lambda^{2} / \hbar^{2}$, and is given by,

$$
E_{m i n}=\frac{\hbar^{2} k_{R}^{2}}{2 m}-\frac{m \lambda^{2}}{2 \hbar^{2}}-\frac{\hbar^{2} h^{2}}{2 m \lambda^{2}}=-\frac{\hbar^{2} h^{2}}{2 m \lambda^{2}} .
$$

\section{B. Two-body bound state}

The two-body problem with zero COM momentum in a uniform system has already been solved in [27]. However, real experiments are carried out well above the critical temperature [6, 7], where two-particle states with a non-zero COM momentum may become important. As we shall see, at the quantitive level, molecules with a nonzero COM momentum can have small contribution to the (momentum-resolved) rf spectroscopy. Here, we outline briefly the general solution with arbitrary COM momenta.

Under the gauge transformation for the field operators, the form of the interatomic interaction (2) is invariant, $\mathcal{H}_{i n t}=U_{0} \int d \mathbf{r} \psi_{\uparrow}^{\dagger}(\mathbf{r}) \psi_{\downarrow}^{\dagger}(\mathbf{r}) \psi_{\downarrow}(\mathbf{r}) \psi_{\uparrow}(\mathbf{r})$. For a two-body problem in the presence of SO coupling, the COM momentum is a good quantum number, and therefore the two-body wavefunction at a given COM momentum $\mathbf{q}$ can generally be written as (we set the volume $V=1$ ) 26]

$$
\begin{aligned}
\left|\Psi_{2 B}(\mathbf{q})\right\rangle=\frac{1}{\sqrt{2 \mathcal{C}}} \sum_{\mathbf{k}}\left[\psi_{\uparrow \downarrow}(\mathbf{k}) c_{\mathbf{q} / 2+\mathbf{k} \uparrow}^{\dagger} c_{\mathbf{q} / 2-\mathbf{k} \downarrow}^{\dagger}+\psi_{\downarrow \uparrow}(\mathbf{r}) c_{\mathbf{q} / 2+\mathbf{k} \downarrow}^{\dagger} c_{\mathbf{q} / 2-\mathbf{k} \uparrow}^{\dagger}+\right. & \\
& \left.\psi_{\uparrow \uparrow}(\mathbf{k}) c_{\mathbf{q} / 2+\mathbf{k} \uparrow}^{\dagger} c_{\mathbf{q} / 2-\mathbf{k} \uparrow}^{\dagger}+\psi_{\downarrow \downarrow}(\mathbf{k}) c_{\mathbf{q} / 2+\mathbf{k} \downarrow}^{\dagger} c_{\mathbf{q} / 2-\mathbf{k} \downarrow}^{\dagger}\right]|v a c\rangle,
\end{aligned}
$$

where $c_{\mathbf{k} \uparrow}^{\dagger}$ and $c_{\mathbf{k} \downarrow}^{\dagger}$ are creation field operators of spin-up and spin-down atoms with momentum $\mathbf{k}$, and $\mathcal{C}=$ $\sum_{\mathbf{k}}\left[\left|\psi_{\uparrow \downarrow}(\mathbf{k})\right|^{2}+\left|\psi_{\downarrow \uparrow}(\mathbf{k})\right|^{2}+\left|\psi_{\uparrow \uparrow}(\mathbf{k})\right|^{2}+\left|\psi_{\downarrow \downarrow}(\mathbf{k})\right|^{2}\right]$ is the normalization factor of the two-body wavefunction. The Schrödinger equation $\mathcal{H}\left|\Psi_{2 B}(\mathbf{q})\right\rangle=\varepsilon_{B}(\mathbf{q})\left|\Psi_{2 B}(\mathbf{q})\right\rangle$ is equivalent to the following set of coupled equations,

$$
\begin{aligned}
& {\left[\varepsilon_{B}(\mathbf{q})-\left(\frac{\hbar^{2} k_{R}^{2}}{m}+\frac{\hbar^{2} k^{2}}{m}+\frac{\hbar^{2} q^{2}}{4 m}+2 \lambda k_{x}\right)\right] \psi_{\uparrow \downarrow}(\mathbf{k})=+\left(U_{0} / 2\right) \sum_{\mathbf{k}^{\prime}}\left[\psi_{\uparrow \downarrow}\left(\mathbf{k}^{\prime}\right)-\psi_{\downarrow \uparrow}\left(\mathbf{k}^{\prime}\right)\right]+h \psi_{\uparrow \uparrow}(\mathbf{k})+h \psi_{\downarrow \downarrow}\left(\mathbf{k}(2)=-\left(U_{0} / 2\right) \sum_{\mathbf{k}^{\prime}}\left[\psi_{\uparrow \downarrow}\left(\mathbf{k}^{\prime}\right)-\psi_{\downarrow \uparrow}\left(\mathbf{k}^{\prime}\right)\right]+h \psi_{\uparrow \uparrow}(\mathbf{k})+h \psi_{\downarrow \downarrow}\left(\mathbf{k}\left(\frac{\hbar^{2} k_{R}^{2}}{m}+\frac{\hbar^{2} k^{2}}{m}+\frac{\hbar^{2} q^{2}}{4 m}-2 \lambda k_{x}\right)\right] \psi_{\downarrow \uparrow}(\mathbf{k})=h \varepsilon_{B}(\mathbf{q})-\left(\frac{\hbar^{2} k_{R}^{2}}{m}+\frac{\hbar^{2} k^{2}}{m}+\frac{\hbar^{2} q^{2}}{4 m}-\lambda q_{x}\right)\right] \psi_{\uparrow \uparrow}(\mathbf{k})=h \psi_{\uparrow \downarrow}(\mathbf{k})+h \psi_{\downarrow \uparrow}(\mathbf{k})} \\
& {\left[\varepsilon_{B}(\mathbf{q})-\left(\frac{\hbar^{2} k_{R}^{2}}{m}+\frac{\hbar^{2} k^{2}}{m}+\frac{\hbar^{2} q^{2}}{4 m}+\lambda q_{x}\right)\right] \psi_{\downarrow \downarrow}(\mathbf{k})=h \psi_{\uparrow \downarrow}(\mathbf{k})+h \psi_{\downarrow \uparrow}(\mathbf{k}) .}
\end{aligned}
$$

Here, $\varepsilon_{B}(\mathbf{q})$ is the energy of the two-body wavefunction which is dependent on the COM momentum q. By introducing

$$
A_{\mathbf{k q}} \equiv \varepsilon_{B}(\mathbf{q})-\left(\frac{\hbar^{2} k_{R}^{2}}{m}+\frac{\hbar^{2} k^{2}}{m}+\frac{\hbar^{2} q^{2}}{4 m}\right)
$$

$$
\begin{aligned}
\psi_{\uparrow \uparrow}(\mathbf{k})= & \frac{\sqrt{2} h}{A_{\mathbf{k q}}-\lambda q_{x}} \psi_{a}(\mathbf{k}) \\
\psi_{\downarrow \downarrow}(\mathbf{k})= & \frac{\sqrt{2} h}{A_{\mathbf{k q}}+\lambda q_{x}} \psi_{a}(\mathbf{k}) \\
\psi_{a}(\mathbf{k})= & \frac{2 \lambda k_{x}}{4 h^{2}+\lambda^{2} q_{x}^{2}} \times \\
& \left(\frac{\lambda^{2} q_{x}^{2}}{A_{\mathbf{k q}}}+\frac{4 h^{2} A_{\mathbf{k q}}}{A_{\mathbf{k q}}^{2}-4 h^{2}-\lambda^{2} q_{x}^{2}}\right) \psi_{s}(\mathbf{k})
\end{aligned}
$$$$
\psi_{s}(\mathbf{k})=\left[\psi_{\uparrow \downarrow}(\mathbf{k})-\psi_{\downarrow \uparrow}(\mathbf{k})\right] / \sqrt{2} \text { and } \psi_{a}(\mathbf{k})=\left[\psi_{\uparrow \downarrow}(\mathbf{k})+\right.
$$$$
\left.\psi_{\downarrow \uparrow}(\mathbf{k})\right] / \sqrt{2} \text {, it is easy to show that, }
$$ 
and

$$
\left[A_{\mathbf{k q}}-\frac{4 \lambda^{2} k_{x}^{2} / A_{\mathbf{k q}}}{1-4 h^{2} /\left(A_{\mathbf{k q}}^{2}-\lambda^{2} q_{x}^{2}\right)}\right] \psi_{s}(\mathbf{k})=U_{0} \sum_{\mathbf{k}^{\prime}} \psi_{s}\left(\mathbf{k}^{\prime}\right) .
$$

From Eq. (28), the un-normalized spin-singlet wavefunction $\psi_{s}(\mathbf{k})$ is given by,

$$
\psi_{s}(\mathbf{k})=\left[A_{\mathbf{k q}}-\frac{4 \lambda^{2} k_{x}^{2} / A_{\mathbf{k q}}}{1-4 h^{2} /\left(A_{\mathbf{k q}}^{2}-\lambda^{2} q_{x}^{2}\right)}\right]^{-1} .
$$

Thus, the two-body binding energy is determined by $U_{0} \sum_{\mathbf{k}} \psi_{s}(\mathbf{k})=1$, or

$$
\frac{m}{4 \pi \hbar^{2} a_{s}}-\sum_{\mathbf{k}}\left[\psi_{s}(\mathbf{k})+\frac{m}{\hbar^{2} k^{2}}\right]=0 .
$$

Here, the bare interaction $U_{0}$ has been regularized by the $s$-wave scattering length $a_{s}$. A bound state exists if its energy satisfies $\varepsilon_{B}(\mathbf{q})<2 E_{\min }$, where $E_{\min }$ is the lowest single-atom energy given by Eq. (18). The normalization factor for the wavefunction is given by

$$
\begin{aligned}
\mathcal{C}= & \sum_{\mathbf{k}}\left|\psi_{s}(\mathbf{k})\right|^{2}\left\{1+\left[\frac{4 h^{2}\left(A_{\mathbf{k q}}^{2}+\lambda^{2} q_{x}^{2}\right)}{\left(A_{\mathbf{k q}}^{2}-\lambda^{2} q_{x}^{2}\right)^{2}}+1\right] \times\right. \\
& \left.\frac{4 \lambda^{2} k_{x}^{2} / A_{\mathbf{k q}}^{2}}{\left[1-4 h^{2} /\left(A_{\mathbf{k q}}^{2}-\lambda^{2} q_{x}^{2}\right)\right]^{2}}\right\} .
\end{aligned}
$$

The bound state energy can be easily solved from Eq. (30) and the results are plotted as a function of the $s$ wave scattering length in Fig 1 for zero COM momentum at three typical values of the Zeeman field $\left(h / E_{\lambda}=\right.$ $0.2,0.5,0.8)$. Here, we have introduced $k_{R}=m \lambda / \hbar^{2}$ and $E_{\lambda}=m \lambda^{2} / \hbar^{2}$ as the units of the wavevector and energy, respectively. We find that the bound state only exists for $a_{s}>0$.

Due to the SO coupling, the effective mass of the twobody bound state is affected. In the limit of small COM momentum, i.e., $\mathbf{q} \approx 0$, the energy of two-body bound state would have a well-defined dispersion,

$$
\varepsilon_{B}(\mathbf{q})=\varepsilon_{B}(0)+\frac{\hbar^{2} q_{x}^{2}}{2 M_{x}}+\frac{\hbar^{2} q_{\perp}^{2}}{2 M_{\perp}}
$$

where $\varepsilon_{B}(0)$ is the two-body binding energy with zero COM momentum, and $M_{x, \perp}$ are the effective masses in $x$ and transverse $(y$ and $z$ ) directions, respectively. The transverse effective mass $M_{\perp}$ is not affected by the $\mathrm{SO}$ coupling, i.e., $M_{\perp}=2 m$, since the SO coupling is only applied along $x$ direction, therefore

$$
A_{\mathrm{kq}}=A_{\mathbf{k}}+\frac{\hbar^{2} q_{x}^{2}}{4 m}\left(\frac{2 m}{M_{x}}-1\right)
$$



Figure 1: (Color online) Binding energies $-\varepsilon_{B} / E_{\lambda}$ of two atoms with zero COM momentum $\mathbf{q}=0$ as functions of the $s$ wave scattering length $1 /\left(k_{R} a_{s}\right)$ for three typical values of the Zeeman field. The horizontal dotted lines are the threshold energies $2 E_{\min }$ where the bound states disappear. Here, we have introduced $k_{R}=m \lambda / \hbar^{2}$ and $E_{\lambda}=m \lambda^{2} / \hbar^{2}$ as the units of the wavevector and energy, respectively.

where $A_{\mathbf{k}}=\varepsilon_{B}(0)-\hbar^{2} k_{R}^{2} / m-\hbar^{2} k^{2} / m$. Substituting Eq. (33) into Eq. (30), expanding up to the second order of $q_{x}$, and comparing the corresponding coefficients, we can easily obtain

$$
\frac{1}{\gamma} \equiv \frac{2 m}{M_{x}}=1-\frac{m \lambda^{2}}{\hbar^{2}} \cdot \frac{I_{2}}{I_{1}}
$$

where

$$
\begin{aligned}
& I_{1}=-\sum_{\mathbf{k}} \frac{\left(A_{\mathbf{k}}^{2}-4 h^{2}\right)^{2}+4 \lambda^{2} k_{x}^{2}\left(A_{\mathbf{k}}^{2}+4 h^{2}\right)}{4 A_{\mathbf{k}}^{2}\left(A_{\mathbf{k}}^{2}-4 h^{2}-4 \lambda^{2} k_{x}^{2}\right)^{2}}, \\
& I_{2}=\sum_{\mathbf{k}} \frac{16 \lambda^{2} h^{2} k_{x}^{2}}{A_{\mathbf{k}}^{3}\left(A_{\mathbf{k}}^{2}-4 h^{2}-4 \lambda^{2} k_{x}^{2}\right)^{2}} .
\end{aligned}
$$

The effective mass ratio $\gamma \equiv M_{x} /(2 m)$ as a function of the interaction strength is shown in Fig, 2 for three typical values of the Zeeman field $\left(h / E_{\lambda}=0.2,0.5,0.8\right)$. We find in the BEC limit, the effective mass is independent of the values of the Zeeman field, and $\gamma$ approaches unity, since two atoms are deeply bound and form a tightly bound molecule. When the interaction is tuned towards the resonance, the two-body bound state becomes looser, and the SO coupling will induce a relatively large effective mass. However, the large SO coupling could also destroy the bound state at a critical $s$-wave scattering length as we have seen in Fig.1

\section{RADIO-FREQUENCY SPECTROSCOPY}

In this section, we will firstly present the basic idea of the rf transition based on the Fermi's golden rule, and 


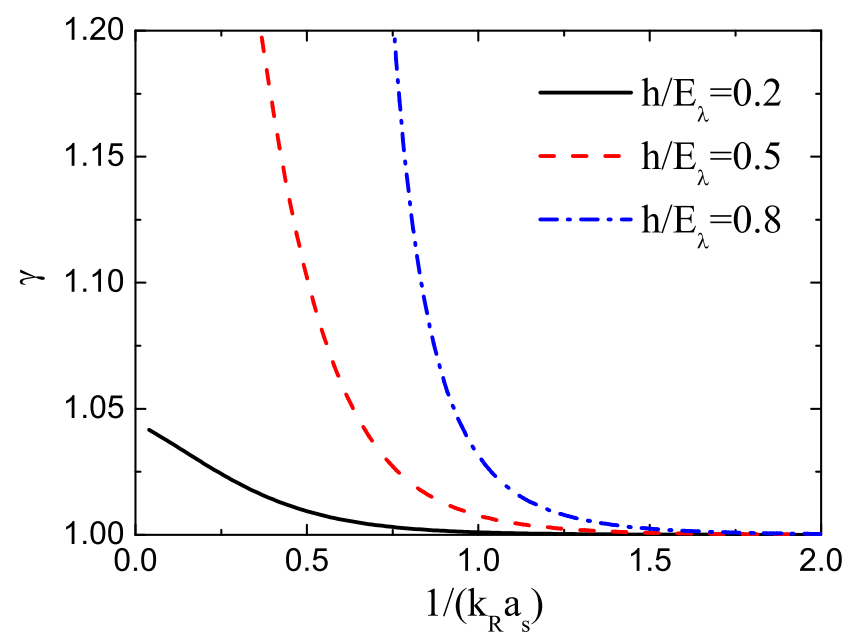

Figure 2: (Color online) The effective mass of weakly bound molecules, $\gamma \equiv M_{x} /(2 m)$, as functions of the interaction strength $1 /\left(k_{R} a_{s}\right)$ for three typical values of the Zeeman field.

then derive the Frank-Condon factor for the rf transition of atoms as well as weakly bound molecules. Finally, under LDA, we theoretically investigate the rf-spectroscopy of a harmonically trapped ideal gas mixture of fermionic atoms and bosonic molecules, which is observable in current experiments.

\section{A. Radio-frequency transition and the Fermi's golden rule}

The basic idear of the rf transition is simple. An rf field is applied to an atomic Fermi gas with two hyperfine states (denoted as $|1\rangle=|\uparrow\rangle$ and $|2\rangle=|\downarrow\rangle$ ), and drives one of the hyperfine states (i.e., $|\downarrow\rangle)$ to an upper state $|3\rangle$ with a bare atomic hyperfine energy difference $\hbar \omega_{3 \downarrow}$ due to the magnetic field splitting. The Hamiltonian of this rf coupling may be written as,

$$
\mathcal{V}_{r f}=V_{0} \int d \mathbf{r}\left[\psi_{3}^{\dagger}(\mathbf{r}) \Psi_{\downarrow}(\mathbf{r})+H . c .\right],
$$

where $\psi_{3}^{\dagger}(\mathbf{r})$ creates an atom in the third state at position $\mathbf{r}$, and $V_{0}$ is the strength of the rf drive and is related to the Rabi frequency $\omega_{R}$ by $V_{0}=\hbar \omega_{R} / 2$. Using the field operator after the gauge transformation, the rf coupling is

$$
\mathcal{V}_{r f}=V_{0} \int d \mathbf{r}\left[e^{-i k_{R} x} \psi_{3}^{\dagger}(\mathbf{r}) \psi_{\downarrow}(\mathbf{r})+\text { H.c. }\right],
$$

or in the momentum space,

$$
\mathcal{V}_{r f}=V_{0} \sum_{\mathbf{k}}\left(c_{\mathbf{k}-k_{R} \mathbf{e}_{x}, 3} c_{\mathbf{k} \downarrow}+\text { H.c. }\right)
$$

for later convenience. Note that, because of the transformation, effectively there is a momentum loss of $k_{R} \mathbf{e}_{x}$ for the transferred atoms.
According to the Fermi's golden rule, the general strength of the rf transition is proportional to the FranckCondon factor [31],

$$
\Gamma(\Omega)=\left|\left\langle\psi_{f}\left|\mathcal{V}_{r f}\right| \psi_{i}\right\rangle\right|^{2} \delta\left(\Omega-\frac{E_{f}-E_{i}}{\hbar}\right),
$$

where $\left|\psi_{i, f}\right\rangle$ denote the initial and final states with corresponding energy $E_{i, f}$, respectively, and $\Omega$ is the frequency of the rf field. The delta function keeps the energy conserved during the transition.

\section{B. The single-particle rf transition}

In the single-particle picture, the eigenstates of a $\mathrm{SO}$ coupled atom are the helicity states $|\mathbf{k}+\rangle(|\mathbf{k}-\rangle)$, other than the original spin states $|\mathbf{k} \uparrow\rangle(|\mathbf{k} \downarrow\rangle)$. If an atom is initially prepared in the state $|\mathbf{k}-\rangle$ with energy $E_{\mathbf{k}--}$, the rf photon will transfer this atom to a third empty state $|3\rangle$. In order to obtain the final state of the rf transition, it's useful to calculate $\mathcal{V}_{r f}|\mathbf{k}-\rangle$. Using Eqs. (17) and (39), we can easily obtain

$$
\mathcal{V}_{r f}|\mathbf{k}-\rangle=V_{0} \cdot \cos \theta_{\mathbf{k}} c_{\mathbf{k}-k_{R} \mathbf{e}_{x}, 3}|v a c\rangle .
$$

This means after the rf transition, there is a probability of $\left|\cos \theta_{\mathbf{k}}\right|^{2}$ that transfers the atom to the final state $|3\rangle$ with a momentum $\mathbf{k}-k_{R} \mathbf{e}_{x}$. Similarly, the final state should be

$$
\mathcal{V}_{r f}|\mathbf{k}+\rangle=V_{0} \cdot \sin \theta_{\mathbf{k}} c_{\mathbf{k}-k_{R} \mathbf{e}_{x}, 3}^{\dagger}|v a c\rangle,
$$

if the atom occupies the state $|\mathbf{k}+\rangle$ before the rf transition. It also means the probability that transfers an atom to the final state $|3\rangle$ with a momentum $\mathbf{k}-k_{R} \mathbf{e}_{x}$ is $\left|\sin \theta_{\mathbf{k}}\right|^{2}$. Generally, the atoms are initially prepared in the mixture states of $|\mathbf{k}-\rangle$ and $|\mathbf{k}+\rangle$ with the probability $f\left(E_{\mathbf{k} \pm}-\mu\right)$, respectively, where $f\left(E_{\mathbf{k} \pm}-\mu\right)$ is the Fermi-Dirac distribution, and $\mu$ is the chemical potential. According to the Fermi's golden rule, the total rf transition strength therefore is given by $\left(V_{0}=1\right)$

$$
\begin{aligned}
\Gamma_{A}(\Omega)= & \sum_{\mathbf{k}} \sin ^{2} \theta_{\mathbf{k}} f\left(E_{\mathbf{k}+}-\mu\right) \delta\left(\Omega-\frac{\Delta \epsilon_{+}}{\hbar}\right)+ \\
& \sum_{\mathbf{k}} \cos ^{2} \theta_{\mathbf{k}} f\left(E_{\mathbf{k}-}-\mu\right) \delta\left(\Omega-\frac{\Delta \epsilon_{-}}{\hbar}\right),(
\end{aligned}
$$

where

$$
\begin{aligned}
\Delta \epsilon_{ \pm} & =\hbar \omega_{3 \downarrow}+\frac{\hbar^{2}\left(\mathbf{k}-k_{R} \mathbf{e}_{x}\right)^{2}}{2 m}-E_{\mathbf{k} \pm} \\
& =-\lambda k_{x} \mp \sqrt{h^{2}+\lambda^{2} k_{x}^{2}}
\end{aligned}
$$

is the probable energy difference between the final and initial states. Without any confusion, we have ignored the bare hyperfine splitting, i.e., $\omega_{3 \downarrow}=0$. 


\section{Two-body bound to free radio-frequency transition}

The picture of the two-body bound to free rf transition is that the rf photon breaks a weakly bound molecule and transfers one of the two atoms to the third state $|3\rangle$. The final state is determined by [27]

$$
\mathcal{V}_{r f}\left|\Psi_{2 B}(\mathbf{q})\right\rangle=V_{0} \sum_{\mathbf{k}} c_{\mathbf{k}-k_{R} \mathbf{e}_{x}, 3}^{\dagger} c_{\mathbf{k}^{\prime} \downarrow}\left|\Psi_{2 B}(\mathbf{q})\right\rangle .
$$

Substituting the wavefunction of the two-body bound state (19) into Eq. (45), and after some straightforward algebra, we shall easily obtain

$$
\begin{gathered}
\mathcal{V}_{r f}\left|\Psi_{2 B}(\mathbf{q})\right\rangle=-\sqrt{\frac{1}{\mathcal{C}}} V_{0} \sum_{\mathbf{k}} c_{\mathbf{q} / 2-\mathbf{k}-k_{R} \mathbf{e}_{x}, 3}^{\dagger} \times \\
\left\{\left[\psi_{s}(\mathbf{k})+\psi_{a}(\mathbf{k})\right] c_{\mathbf{q} / 2+\mathbf{k} \uparrow}^{\dagger}+\sqrt{2} \psi_{\downarrow \downarrow}(\mathbf{k}) c_{\mathbf{q} / 2+\mathbf{k} \downarrow}^{\dagger}\right\}|v a c\rangle .
\end{gathered}
$$

Using Eq. (17), the final state can be written in terms of the helicity basis as

$$
\begin{aligned}
& \mathcal{V}_{r f}\left|\Psi_{2 B}(\mathbf{q})\right\rangle=-\sqrt{\frac{1}{\mathcal{C}}} V_{0} \sum_{\mathbf{k}} c_{\mathbf{q} / 2-\mathbf{k}-k_{R} \mathbf{e}_{x}, 3}^{\dagger} \times \\
& \left(s_{\mathbf{q} / 2+\mathbf{k}+} c_{\mathbf{q} / 2+\mathbf{k}+}^{\dagger}-s_{\mathbf{q} / 2+\mathbf{k}-} c_{\mathbf{q} / 2+\mathbf{k}-}^{\dagger}\right)|v a c\rangle,
\end{aligned}
$$

where

$$
\begin{aligned}
s_{\mathbf{q} / 2+\mathbf{k}+}= & {\left[\psi_{s}(\mathbf{k})+\psi_{a}(\mathbf{k})\right] \cos \theta_{\mathbf{q} / 2+\mathbf{k}}+} \\
& \sqrt{2} \psi_{\downarrow \downarrow}(\mathbf{k}) \sin \theta_{\mathbf{q} / 2+\mathbf{k}}, \\
s_{\mathbf{q} / 2+\mathbf{k}-}= & {\left[\psi_{s}(\mathbf{k})+\psi_{a}(\mathbf{k})\right] \sin \theta_{\mathbf{q} / 2+\mathbf{k}}-} \\
& \sqrt{2} \psi_{\downarrow \downarrow}(\mathbf{k}) \cos \theta_{\mathbf{q} / 2+\mathbf{k}} .
\end{aligned}
$$

Eq. (47) could be understood as follows. During the rf transition, a molecule with COM momentum $\mathbf{q}$ is broken by absorbing the rf photon, and then the spin-down atom is transferred to the third state. Finally, there are two possibilities. We may have two atoms in the third state and upper helicity state, respectively, with a probability $\left|s_{\mathbf{q} / 2+\mathbf{k}+}\right|^{2} / \mathcal{C}$. Also, we may have a probability of $\left|s_{\mathbf{q} / 2+\mathbf{k}-}\right|^{2} / \mathcal{C}$ for having two atoms in the third state and lower helicity state, respectively. Taking into account these two final states, we should have the following transfer strength or the Franck-Condon factor, according to the Fermi's golden rule $\left(V_{0}=1\right)$,

$$
\begin{aligned}
\Gamma_{M}(\Omega)= & \frac{1}{\mathcal{C}} \sum_{\mathbf{k}}\left|s_{\mathbf{q} / 2+\mathbf{k}+}\right|^{2} \delta\left(\Omega-\frac{\Delta \varepsilon_{+}}{\hbar}\right)+ \\
& \frac{1}{\mathcal{C}} \sum_{\mathbf{k}}\left|s_{\mathbf{q} / 2+\mathbf{k}-}\right|^{2} \delta\left(\Omega-\frac{\Delta \varepsilon_{-}}{\hbar}\right)
\end{aligned}
$$

where

$$
\Delta \varepsilon_{ \pm}=\frac{\hbar^{2}\left(\mathbf{q} / 2-\mathbf{k}-k_{R} \mathbf{e}_{x}\right)^{2}}{2 m}+E_{\mathbf{q} / 2+\mathbf{k}, \pm}-\varepsilon_{B}(\mathbf{q})
$$

is the probable energy difference between the final and initial states. Here, we have already ignored the bare hyperfine splitting, i.e., $\omega_{3 \downarrow}=0$ as well. Eqs.(50) and (51) recover the results of [27] when $\mathbf{q}=0$.

\section{Rf spectroscopy for a harmonically trapped gas mixture}

In this subsection, we are ready to investigate theoretically the rf response signals of the harmonically trapped ideal gas mixture of the atoms and molecules, as well as the total rf spectroscopy.

\section{The signals of the atomic component}

The rf transition strength for atoms in a uniform system is determined by Eq. (43). In the harmonic trap, the rf transition strength of atoms at the position $\mathbf{r}$ should be $\Gamma_{A}(\Omega, \mathbf{r})$, in which we shall use the local chemical potential,

$$
\mu(\mathbf{r})=\mu-\frac{m}{2}\left(\omega_{x}^{2} x^{2}+\omega_{y}^{2} y^{2}+\omega_{z}^{2} z^{2}\right),
$$

where $\mu$ is the chemical potential in the trap center determined by Eq. (13). Thus, using LDA the total rf transition strength of the atomic component in a harmonic trap is $\Gamma_{A}(\Omega)=\int d \mathbf{r} \Gamma_{A}(\Omega, \mathbf{r})$.

In the actual calculation, the summation $\sum_{\mathbf{k}}$ in $\Gamma_{A}(\Omega, \mathbf{r})$ may be replaced by the integral $(2 \pi)^{-3} \int d k_{x} \int d^{2} \mathbf{k}_{\perp}$. After some tedious but straightforward derivation, the total atomic rf transition strength has the following form,

$$
\begin{aligned}
\frac{E_{\lambda} \Gamma_{A}(\Omega)}{N}= & -\frac{3}{2 \sqrt{2 \pi}} \cdot\left(\frac{E_{\lambda}}{E_{F}} \cdot \frac{T}{T_{F}}\right)^{3 / 2} \frac{h^{2}}{\Omega^{2}} \times \\
& \int_{0}^{\infty} d k_{\perp}^{2} L i_{3 / 2}\left[-z_{A} e^{-E_{k_{\perp}} /\left(k_{B} T\right)}\right],
\end{aligned}
$$

where

$$
E_{k_{\perp}}=\frac{\hbar^{2}\left(k_{\perp}^{2}+k_{R}^{2}\right)}{2 m}+\frac{\hbar^{2}}{2 m \lambda^{2}}\left(\frac{h^{2}-\Omega^{2}}{2 \Omega}\right)^{2}-\frac{h^{2}+\Omega^{2}}{2 \Omega},
$$

and $E_{F}=\hbar \omega(3 N)^{1 / 3}$ and $T_{F}=E_{F} / k_{B}$ are the Fermi energy and Fermi temperature, respectively. At a given temperature $T$ and interaction strength $E_{B}$, the atomic chemical potential $\mu$ is obtained from Eq. (13). Then, with a fixed total atom number $N$ (which in turn gives the Fermi energy $E_{F}$ ), the rf transition strength of the atomic component can numerically be calculated from Eq. (53). The results are presented in Fig. 3 for different values of Zeeman field at the given parameters, 


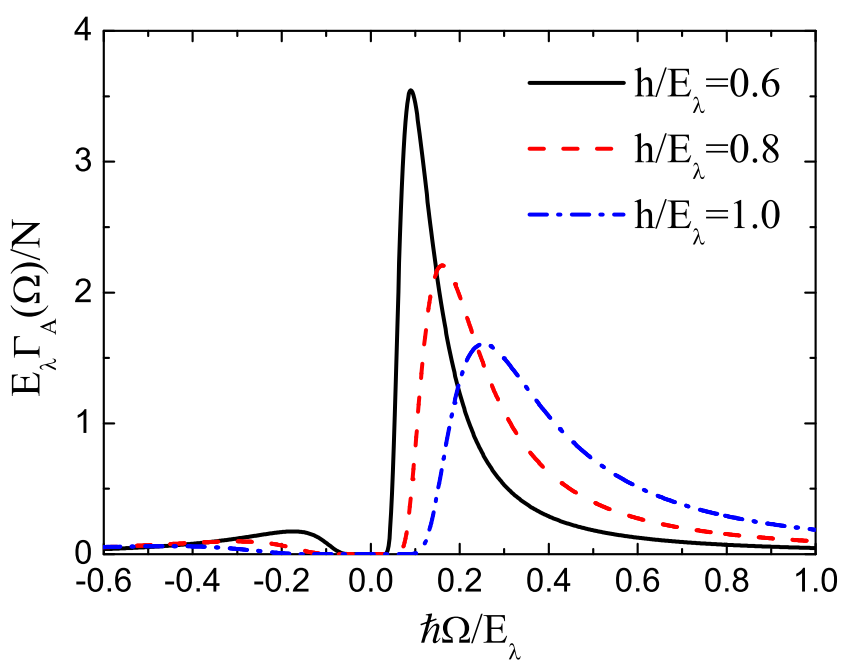

Figure 3: (Color online) The rf spectroscopy of the atomic component in a harmonic trap at different values of the Zeeman field, i.e., $h / E_{\lambda}=0.6,0.8,1.0$. Here, we have taken $E_{B} / E_{\lambda}=2, E_{F} / E_{\lambda}=1$, and $T / T_{F}=1$.
$E_{B} / E_{\lambda}=2, E_{F} / E_{\lambda}=1$ and $T / T_{F}=1$. We find that there is a bimodal structure in the rf spectroscopy of the atomic component. The stronger rf signals at the positive frequency $\Omega$ correspond to the transition out of the lower helicity state, while those with weaker strength at the negative frequency correspond to the transition out of the upper helicity state. On the other hand, there is a red-shift in the peak positions as the strength of the Zeeman field decreases. As we may anticipate, the peak transition frequency $\Omega$ will reach the bare hyperfine splitting $\omega_{3 \downarrow}=0$ in the absence of SO coupling.

In addition to the total strength of the atomic rf transition, we can also calculate the transition strength of atoms at a specific momentum $k_{x}$. This momentumresolved rf spectroscopy has already been investigated at Shanxi University [6] and at MIT [7]. According to Eq. (43), we can carry out the integral over $\mathbf{k}_{\perp}$ and retain that of $k_{x}$. Therefore, the momentum-resolved rf transition strength is determined by

$$
\begin{aligned}
\frac{k_{R} E_{\lambda} \Gamma_{A}\left(\Omega, k_{x}\right)}{N}= & -\frac{3 \sqrt{\pi}}{2 \sqrt{2}}\left(\frac{E_{\lambda}}{E_{F}} \cdot \frac{T}{T_{F}}\right)^{3 / 2}\left(1-\frac{\lambda k_{x}}{\sqrt{h^{2}+\lambda^{2} k_{x}^{2}}}\right) \delta\left(\Omega-\frac{\Delta \epsilon_{+}}{\hbar}\right) \int_{0}^{\infty} d k_{\perp}^{2} L i_{3 / 2}\left(-z_{A} e^{-E_{\mathbf{k}+} / k_{B} T}\right)+ \\
& -\frac{3 \sqrt{\pi}}{2 \sqrt{2}}\left(\frac{E_{\lambda}}{E_{F}} \cdot \frac{T}{T_{F}}\right)^{3 / 2}\left(1+\frac{\lambda k_{x}}{\sqrt{h^{2}+\lambda^{2} k_{x}^{2}}}\right) \delta\left(\Omega-\frac{\Delta \epsilon_{-}}{\hbar}\right) \int_{0}^{\infty} d k_{\perp}^{2} L i_{3 / 2}\left(-z_{A} e^{-E_{\mathbf{k}-} / k_{B} T}\right)(55)
\end{aligned}
$$

Corresponding to experiments, the delta function in Eq. (55) can be replaced by the Lorentz-line shape as,

$$
\delta(x) \rightarrow \frac{1}{\pi} \cdot \frac{\sigma}{x^{2}+\sigma^{2}},
$$

where $\sigma$ is the line width.

Fig. 4- reports the momentum-resolved rf spectroscopy of the atomic component for different values of the Zeeman field and interaction strength. Here, we define $K_{x}=k_{x}-k_{R}$ to make sure the spectra is a symmetric function of $K_{x}$ in the absence of SO coulping [30]. As we anticipate, there are two branches in which one is relatively weaker than the other one. Since the energy of the upper helicity state $|\mathbf{k}+\rangle$ is larger than that of the lower helicity state $|\mathbf{k}-\rangle$, the atoms will occupy the lower helicity state first and then the upper one before the $\mathrm{rf}$ transition. Thus, the initial atomic population of the lower helicity state should be larger. Therefore, similar to the integrated rf spectroscopy, the brighter branch of the momentum-resolved rf spectroscopy corresponds to the rf transition out of the state $|\mathbf{k}-\rangle$, while the weaker one corresponds to the rf transition out of the state $|\mathbf{k}+\rangle$. Therefore, the contribution from two initial states could be well identified experimentally. We can also see that the gap between two branches becomes larger when the strength of the Zeeman field increases. This is because the transition frequency deviates from the bare hyperfine splitting $\omega_{3 \downarrow}$ more obviously with a stronger SO coupling.

All these features, discussed in the above, are in good agreement with the experimental observation for a noninteracting spin-orbit coupled atomic Fermi gas; see, for example, Fig. 4 in Ref. [6] and Fig. 2 in Ref. [7].

\section{The rf spectroscopy of the molecular component}

With the two-body binding energy solved from Eq. (30) at a given COM momentum $\mathbf{q}$, the rf transition strength of a single molecule can be calculated directly from Eq. (50). We plot the rf response of a single molecule with zero COM momentum, as well as nonzero COM momentum (i.e., $q_{x} / k_{R}=0.5$ ) in Fig. 5, Here, the interatomic interaction strength, denoted by $E_{B} / E_{\lambda}=\left[\hbar^{2} /\left(m \lambda a_{s}\right)\right]^{2}$, is set to be unity. Since the SO coupling is only along the $x$ direction, we can set the transverse $\mathrm{COM}$ momentum $\mathbf{q}_{\perp}=0$, which is physically irrelevant and arbitrary. From Fig. 5] we find that there is a slight red-shift in the peak position with a non-zero 




Figure 4: (Color online) The momentum-resolved rf spectroscopy of the harmonically trapped atomic component for different values of the Zeeman field and interaction strength. Here, we have taken $E_{F} / E_{\lambda}=2, T / T_{F}=1$ and $\sigma=0.1$. In addition, we have also defined $K_{x}=k_{x}-k_{R}$ (see text), which moves the whole spectra to the right by an amount $k_{R}$.

COM momentum. This is because the energy gap between the final and initial states becomes smaller at nonzero COM momentum, and consequently a little higher transition peak appears.

The momentum-resolved rf spectroscopy of a single molecule can be obtained from Eq. (50) by integrating over $\mathbf{k}_{\perp}$, and is given by

$$
\begin{array}{r}
\Gamma_{M}\left(\Omega, k_{x}\right)=\frac{m}{8 \pi^{2} \hbar \mathcal{C}}\left[\left|s_{\mathbf{q} / 2+\mathbf{k}+}\right|^{2} \Theta\left(k_{\perp,+}^{2}\right)+\right. \\
\left.\left|s_{\mathbf{q} / 2+\mathbf{k}-}\right|^{2} \Theta\left(k_{\perp,-}^{2}\right)\right],
\end{array}
$$

where $\Theta(x)$ is the step function and

$$
\begin{aligned}
& k_{\perp, \pm}^{2}=\frac{m}{\hbar}\left[\Omega+\varepsilon_{B}(\mathbf{q})\right]-\left[\frac{k_{x}^{2}+\left(k_{x}+k_{R}\right)^{2}}{2}+\right. \\
& \left.\frac{k_{R}^{2}+\left(k_{R}+q_{x}\right)^{2}}{4} \pm \frac{m}{\hbar^{2}} \sqrt{h^{2}+\lambda^{2}\left(\frac{q_{x}}{2}+k_{x}\right)^{2}}\right] .
\end{aligned}
$$



Figure 5: (Color online) The rf spectroscopy of a single molecule for different values of the Zeeman field at given COM momenta $\mathbf{q} / k_{R}=0$ and $\mathbf{q} / k_{R}=0.5$. Here, we have chosen an interaction strength $E_{B} / E_{\lambda}=1$.

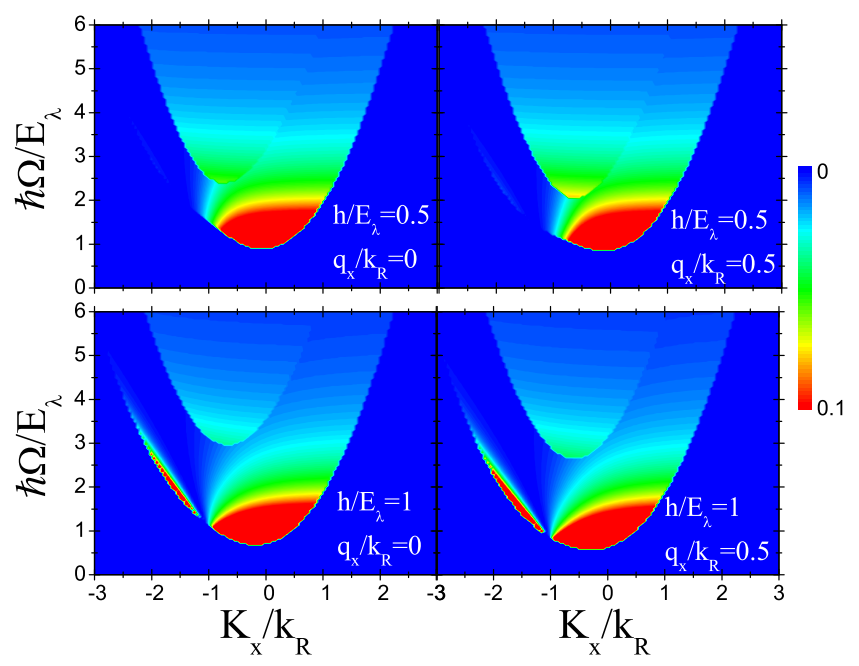

Figure 6: (Color online) The momentum-resolved rf spectroscopy of a single molecule for different values of the Zeeman field at given COM momenta $\mathbf{q}_{x} / k_{R}=0$ and $\mathbf{q} / k_{R}=0.5$. Here, we have chosen the interaction strength $E_{B} / E_{\lambda}=1$.

The momentum-resolved rf spectroscopy of a single molecule with zero and non-zero COM momentum $\left(q_{x} / k_{R}=0.5\right)$ are presented in Fig. 6. In this plot, we have defined $K_{x}=-k_{x}-k_{R}$, as we require that in the absence of SO coupling the spectra is an even function of $K_{x}$ [27]. We find that the contribution from two final states are well separated in different frequency domain and therefore should be easily observed experimentally.

\section{The total rf spectroscopy of the gas mixture of atoms and molecules}

By increasing the attractive interaction strength of a two-component Fermi gas at finite temperatures, the system will evolve into a mixture of fermionic atoms and bosonic molecules. We have so far discussed the integrated and momentum-resolved rf responses for both atoms and molecules. The total rf spectroscopy of a 


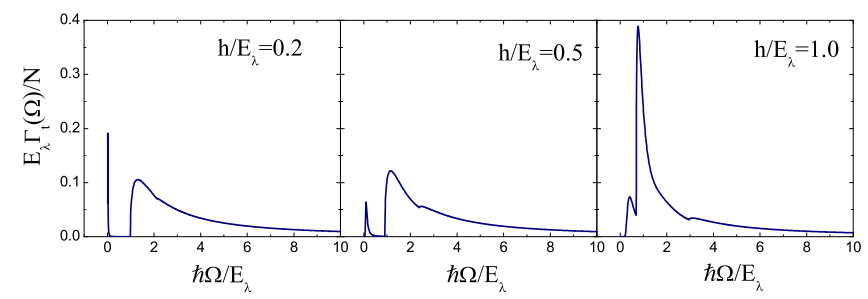

Figure 7: (Color online) The total rf spectroscopy of a harmonically trapped ideal gas mixture of fermionic atoms and bosonic molecules at different Zeeman field. Here, we take $E_{B} / E_{\lambda}=1, E_{F} / E_{\lambda}=1$ and $T / T_{F}=0.2$.

harmonically trapped ideal gas mixture of atoms and molecules can now be easily obtained.

For the atomic component, the rf transition strength is already given by Eq. (53). As to the molecules, the population $N_{M}$ can be calculated from Eq. (11) at given temperature $T / T_{F}$ and interaction strength $E_{B} / E_{\lambda}$. In real experiments, the temperature is low so that the distribution of the molecular COM momentum $\mathbf{q}$ is very narrow around $\mathbf{q}=0$. As already seen from Figs. 5 and 6 , the rf spectroscopy of molecules depends weakly on q. Thus, as a good qualitative approximation, we may focus on the rf transition of molecules with zero COM momentum only. Then, the total rf transition strength of molecular component is given by $N_{M} \Gamma_{M}(\Omega)$. In the end, we obtain the total rf spectroscopy,

$$
\Gamma_{t}(\Omega)=\Gamma_{A}(\Omega)+N_{M} \Gamma_{M}(\Omega) .
$$

Fig. 7 shows the total rf spectroscopy of the atommolecule mixture at different Zeeman field and at fixed temperature $T / T_{F}=0.2$ and interaction strength $E_{B} / E_{\lambda}=1$. It is obvious that there is a double-peak structure in the spectroscopy. The peak at higher frequency is responsible for weakly bound molecules, since more energy is required for pair breaking. With increasing the Zeeman field $h$, the energy difference between the final and initial states of atoms during the rf transition becomes larger, thus a decrease in the atomic peak is observed. On the other hand, since the energy difference between the molecular final and initial states becomes smaller as the Zeeman field increases, the rf signal of molecules grows, as we may anticipate.

In order to show the temperature dependence, we also calculate the total rf response of the ideal gas mixture at different temperatures and at a fixed Zeeman field $h / E_{\lambda}=0.5$, as reported in Fig. 8. As the temperature decreases, the population of molecules becomes larger. Thus, a reduction of the atomic signal is observed. The molecular response should dominate when temperature decreases to zero.

Finally, we present the total momentum-resolved rf spectroscopy in Fig. 9, with parameters correpsonding to Fig. 7 (see the left panel) and Fig. 8 (right panel).

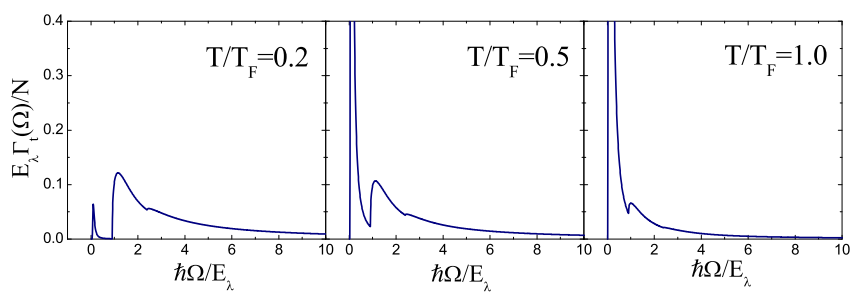

Figure 8: (Color online) The total rf spectroscopy of a harmonically trapped ideal gas mixture of fermionic atoms and bosonic molecules as a function of temperature at a fixed Zeeman field strength $h / E_{\lambda}=0.5$. Here, we use $E_{B} / E_{\lambda}=1$ and $E_{F} / E_{\lambda}=1$.

\section{CONCLUSIONS}

In conclusion, we have theoretically investigated the momentum-resolved radio-frequency spectroscopy of a harmonically trapped ideal gas mixture of fermionic atoms and bosonic molecules with spin-orbit coupling. This is a qualitative theoretical model of spin-orbit coupled atomic Fermi gases near broad Feshbach resonances.

In particular, for local uniform systems, general formulas for the wavefunction and binding energy of weakly bound bosonic molecules with non-zero center-of-mass momentum have been derived. The influence of this nonzero center-of-mass momentum on the radio-frequency spectroscopy of molecules has been discussed in detail.
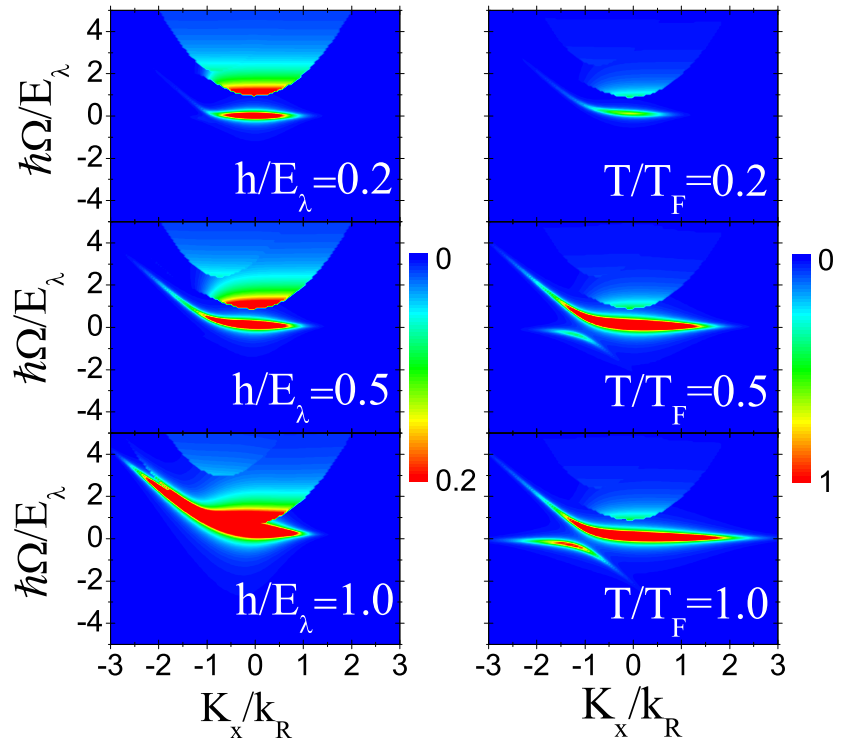

Figure 9: (Color online) The total momentum-resolved rf spectroscopy of a harmonically trapped ideal gas mixture of fermionic atoms and bosonic molecules. The left panel shows the spectra at different Zeeman field strength at a fixed temperature $T / T_{F}=0.2$, while the right panel shows the temperature dependence of spectroscopy at a fixed Zeeman field $h / E_{\lambda}=0.5$. Here, we take $E_{B} / E_{\lambda}=1$ and $E_{F} / E_{\lambda}=1$. Note the different color scale in the left and right panels. 
Based on few-body solutions in uniform systems, the radio-frequency responses of atomic and molecular components in harmonic traps have been calculated within local density approximation, respectively, by using Fermi's golden rule. Our results provide a qualitative picture of momentum-resolved radio-frequency spectroscopy of a strongly interacting spin-orbit coupled Fermi gas in harmonic traps, which could be directly observed in future experiments at Shanxi University [6, 20] and MIT[7, 21].

In real experiments, where strongly interacting Fermi gases are created in the vicinity of Feshbach resonances, the atoms and weakly bound molecules must strongly interact with each other. An in-depth analysis of radiofrequency spectroscopy would rely on much more complicated many-body theories 32], beyond the simple few- body picture presented in this work.

\section{Acknowledgments}

Shi-Guo Peng and Kaijun Jiang are supported by China Postdoctoral Science Foundation (Grant No. 2012M510187), the NSFC projects (Grant No. 11004224 and No.11204355) and the NFRP-China (Grant No. 2011CB921601). Xia-Ji Liu and Hui Hu are supported by the ARC Discovery Projects (Grant No. DP0984637 and No. DP0984522) and the NFRP-China (Grant No. 2011CB921502).
[1] I. Bloch, J. Dalibard, and W. Zwerger, Rev. Mod. Phys. 80, 885 (2008).

[2] S. Giorgini, L. P. Pitaevskii, and S. Stringari, Rev. Mod. Phys. 80, 1215 (2008).

[3] C. Chin, R. Grimm, P. S. Julienne, and E. Tiesinga, Rev. Mod. Phys. 82, 1225 (2010).

[4] Y. J. Lin, R. L. Compton, K. Jimenez-Garcia, W. D. Phillips, J. V. Porto, and I. B. Spielman, Nat. Physics 7, 531 (2011).

[5] Y. J. Lin, K. Jimenez-Garcia, and I. B. Spielman, Nature (London) 471, 83 (2011).

[6] P. Wang, Z.-Q. Yu, Z. Fu, J. Miao, L. Huang, S. Chai, H. Zhai, and J. Zhang, Phys. Rev. Lett. 109, 095301 (2012).

[7] L. W. Cheuk, A. T. Sommer, Z. Hadzibabic, T. Yefsah, W. S. Bakr, and M. W. Zwierlein, Phys. Rev. Lett. 109, 095302 (2012).

[8] J. Dalibard, F. Gerbier, G. Juzeliunas, and P. Ohberg, Rev. Mod. Phys. 83, 1523 (2011).

[9] X.-L. Qi and S.-C. Zhang, Physics Today 63, 33 (2010).

[10] M. Z. Hasan and C. L. Kane, Rev. Mod. Phys. 82, 3045 (2010).

[11] H. Zhai, Int. J. Mod. Phys. B 26, 1230001 (2012).

[12] C. Chin, M. Bartenstein, A. Altmeyer, S. Riedl, S. Jochim, J.H. Denschlag, and R. Grimm, Science 305, 1128 (2004).

[13] C.H. Schunck, Y.I. Shin, A. Schirotzek, and W. Ketterle, Nature (London) 454, 739 (2008) .

[14] A. Schirotzek, C. H. Wu, A. Sommer, and M. W. Zwierlein, Phys. Rev. Lett. 102, 230402 (2009).

[15] Y. Zhang, W. Ong, I. Arakelyan, J.E. Thomas, Phys. Rev. Lett. 108, 235302 (2012).
[16] M. Koschorreck, D. Pertot, E. Vogt, B. Frohlich, M. Feld, and M. Kohl, Nature (London) 485, 619 (2012).

[17] C. Kohstall, M. Zaccanti, M. Jag, A. Trenkwalder, P. Massignan, G. M. Bruun, F. Schreck, and R. Grimm, Nature (London) 485, 615 (2012).

[18] C. A. Regal, C. Ticknor, J. L. Bohn, and D. S. Jin, Nature (London) 424, 47 (2003).

[19] J. T. Stewart, J. P. Gaebler, and D. S. Jin, Nature (London) 454, 744 (2008).

[20] J. Zhang, private communication.

[21] M. Zwierlein, private communication.

[22] H. Hu, X.-J. Liu, and P. D. Drummond, Phys. Rev. A 77, 061605(R) (2008).

[23] H. Hu, X.-J. Liu, and P. D. Drummond, New J. Phys. 12, 063038 (2010).

[24] J. P. Vyasanakere and V. B. Shenoy, Phys. Rev. B 83, 094515 (2011).

[25] H. Hu, L. Jiang, X.-J. Liu, and H. Pu, Phys. Rev. Lett. 107, 195304 (2011).

[26] Z. Q. Yu and H. Zhai, Phys. Rev. Lett. 107, 195305 (2011).

[27] H. Hu, H. Pu, J. Zhang, S.-G. Peng, and X.-J. Liu, arXiv: 1208.5841

[28] W. Yi and L. M. Duan, Phys. Rev. A 73, 031604 (2006).

[29] X.-J. Liu, H. Hu, and P. D. Drummond, Phys. Rev. A 75, 023614 (2007).

[30] X.-J. Liu, Phys. Rev. A 86, 033613 (2012).

[31] C. Chin, P. S. Julienne, Phys. Rev. A, 71, 012713 (2005).

[32] H. Hu, X.-J. Liu, and P. D. Drummond, Europhys. Lett. 74, 574 (2006). 